Il ricordo di Amedeo de Vecchi in questo momento della mia vita mi crea notevole difficoltà. Negli ultimi anni dopo il suo pensionamento che tanto lo aveva fatto soffrire, era venuto a collaborare con me in un Centro di emodialisi situato in una RSA. Competente, preparato, molto umano e sensibile si collaborava molto bene, mancherà a tutti noi. La sua competenza in dialisi peritoneale era nota, come confermano i numerosi studi eseguiti; proprio da un lavoro scritto nel 2000 è nato lo spunto per l'articolo comparso sul GIN no. 42010 sulla dialisi peritoneale incrementale che con il mio gruppo ho cercato di elaborare in modo differente. Pertanto partecipo a questo dibattito sulla marginalità della DP inviando un riassunto del lavoro e ricordando che alcuni studi hanno confermato come la 'dialisi 'peritoneale non sia una dialisi marginale ma una soluzione comune per diversi pazienti.

\title{
La dialisi peritoneale incrementale nella qualità e quantità pone la peritoneale un trattamento non marginale
}

\author{
Silvio Volmer Bertoli, Daniele Ciurlino, Andrea Stucchi
}

U.O. Nefrologia e Dialisi, Dipartimento Cardiovascolare, IRCCS Multimedica, Sesto San Giovanni (MI)

\begin{abstract}
INCREMENTAL PERITONEAL DIALYSIS: A NON-MARGINAL TREATMENT, QUALITATIVELY AND QUANTITATIVELY
Abstract. Incremental peritoneal dialysis has been proved feasible and safe in asymptomatic patients with a glomerular filtration rate $(G F R)<6 \mathrm{~mL} / \mathrm{min}$. A second population is composed of asymptomatic, mostly older patients with GFR between 6 and $10 \mathrm{~mL} / \mathrm{min}$, in whom a low-dose start may preserve the residual renal function. Lastly, patients with severe, terminal, chronic cardiomyopathy who are not candidates for a heart transplant may experience beneficial effects on cardiac function and hospitalization with low-dose peritoneal dialysis treatment even when they have GFR $>10 \mathrm{~mL} / \mathrm{min}$. In conclusion, incremental peritoneal dialysis is a feasible therapeutic option that the nephrologist should know and be able to perform in those patients who may benefit from it. Therefore, it is noted that peritoneal dialysis is not a treatment marginal.
\end{abstract}

Key words: Incremental peritoneal dialysis, Cardiac decompensation, Elderly patients

Conflict of interest: None.

Financial support: None.

Accettato: 8 Dicembre 2013

Tra le metodiche dialitiche, la dialisi peritoneale (DP) è quella che si presta più facilmente alla "personalizzazione" del trattamento, sia in termini di soluzioni, che soprattutto di tempi e dosi dialitiche. Il mutamento della popolazione dialitica che si è progressivamente verificato negli ultimi anni ha posto ai Nefrologi nuove e diverse sfide in scenari via via più complessi. La DP può essere avviata con uno schema di dialisi incrementale (DPI), che permette un trattamento con una dose dialitica bassa, che viene poi modificata nel tempo al deterioramento della funzione renale residua (1). Non vi è accordo su quando iniziare la terapia sostitutiva, e le Linee Guida lasciano allo specialista la valutazione dei rischi e dei benefici (2). É ancora motivo di dibattito se la dialisi peritoneale debba essere iniziata con un dosaggio pieno o con dosi incrementali. L'utilizzo di una dialisi a basse dosi non è sempre fattibile, ma potrebbe avere il vantaggio di migliorare la qualità di vita e di ridurre la frequenza di complicanze infettive e metaboliche. Tuttavia, la dialisi peritoneale incrementale deve raggiungere $i$ targets minimi di adeguatezza consigliati, riguardo a depurazione (Kt/V, creatinina clearance), ultrafiltrazione e bilancio elettrolitico. La dialisi peritoneale incrementale è attuabile e sicura in pazienti asintomatici con un filtrato glomerulare $<6 \mathrm{~mL} /$ min, ma con l'obbligo di monitorare attentamente la funzione renale. La DPI può essere proposta a pazienti asintomatici con 
GFR $<6 \mathrm{~mL} / \mathrm{min}$ fintanto che si mantengono i minimi target di adeguatezza dialitica. Inoltre può avere diversi effetti positivi, non ultimo un impatto favorevole sulla scelta della metodica. I risultati positivi ottenuti sul rallentamento della progressione della insufficienza renale con DP potrebbero essere uno stimolo per indagare un inizio più precoce della DPI. Tale metodica sarebbe indicata anche in pazienti asintomatici, anziani, con filtrato glomerulare tra 6 e $10 \mathrm{~mL} / \mathrm{min}$, nei quali l'inizio con una terapia a dosi incrementali potrebbe preservare la funzione renale residua e favorire un training più graduale. Il razionale di questo approccio si basa sul fatto che la morbilità aumenta con il declino della funzione renale e che pertanto anche una depurazione peritoneale ottenuta con DPI è in grado almeno in parte di ridurre il peggioramento delle condizioni cliniche. Un secondo aspetto da tenere in considerazione è che lo stato nutrizionale peggiora con la riduzione della funzione renale e che l'avvio del trattamento sostitutivo in pazienti malnutriti è correlato con una cattiva prognosi. Nella nostra esperienza personale (3), l'avvio della CAPD a basse dosi è stata possibile in 24 pazienti $(M / F=17 / 7$; età: $73 \pm 9$ anni) negli ultimi otto anni, pari al $30 \%$ dei pazienti che hanno iniziato la DP. All'avvio del trattamento i pazienti avevano in media un $\mathrm{Kt} / \mathrm{V}$ settimanale renale di $1.4 \pm 0.4$, un GFR di $9.6 \pm 2.7 \mathrm{~mL} / \mathrm{min}$ e una clearance della creatinina di $14 \pm 4.8 \mathrm{~mL} / \mathrm{min}$ : di questi pazienti 10 hanno iniziato il trattamento con un GFR superiore a $10 \mathrm{~mL} / \mathrm{min}$ per sovraccarico idrico cronico da cardiopatia dilatativa ipocinetica severa, utilizzando icodestrina notturna. Gli altri 14 pazienti hanno iniziato il trattamento con un GFR $<10 \mathrm{~mL} / \mathrm{min}$ ancora paucisintomatici con lo scopo di ottenere un adattamento alla metodica in considerazione della loro età avanzata. Il tempo medio di terapia a basse dosi è stato di 11.0 \pm 7.0 mesi e al termine dell'osservazione (fine 2008) dei 14 pazienti con GFR tra 6 e $10 \mathrm{~mL} / \mathrm{min}, 11$ hanno aumentato la posologia dialitica, mentre 3 sono ancora in "monoscambio". Il monoscambio a questi valori di filtrato in pazienti anziani rappresenta una possibilità di adattamento domiciliare alla metodica in soggetti a rischio di rapido deterioramento della funzione renale per patologie intercorrenti e quindi con gravi difficoltà ad un addestramento dialitico domiciliare. In ultimo, i pazienti con cardiomiopatia cronica severa e terminale non candidati ad un trapianto di cuore, potrebbero avere degli effetti vantaggiosi sulla funzione cardiaca e sull'ospedalizzazione con un trattamento dialitico a basse dosi, anche con GFR $>10 \mathrm{~mL} / \mathrm{min}$. L'obiettivo terapeutico in questi pazienti è duplice: da un lato il mantenimento dell'euvolemia e la prevenzione degli episodi di scompenso acuto, dall'altro il miglioramento della cinetica cardiaca e verosimilmente una migliore prognosi in pazienti già in terapia medica massimale. Spesso questi pazienti beneficiano di un singolo scambio notturno e tale terapia può essere proseguita per lungo tempo anche per una conservazione della funzione renale legata ad un miglioramento della performance cardiaca. Un recente studio retrospettico osservazionale svoltosi in 10 centri nefrologici italiani, in pazienti con scompenso cardiaco severo ha mostrato una riduzione dell'ospedalizzazione, un miglioramento della classe NYHA funzionale ed una sopravvivenza ad un anno dell' $85 \%$ utilizzando l'ultrafiltrazione peritoneale (4). In conclusione, la dialisi peritoneale incrementale è un'opzione terapeutica che il nefrologo dovrebbe conoscere ed essere in grado di attuare in quei pazienti che potrebbero trarne beneficio.

\section{Riassunto}

La DPI rappresenta un'opportunità per favorire il trattamento domiciliare. In questo momento la terapia sostitutiva domiciliare con DP rappresenta una sfida a causa dell'invecchiamento della popolazione e le difficoltà che abbiamo nel preparare tali pazienti a questa terapia. Nel paziente in uremia terminale la DPI rappresenta una opportunità di adattamento alla terapia, non sempre possibile; in caso invece di GFR superiore a $6 \mathrm{~mL} /$ min può rappresentare una possibilità di adattamento più semplice e graduale alla terapia, viste le migliori condizioni cliniche generali. Nel paziente con scompenso cardiaco refrattario riteniamo che sia il trattamento ideale nella gestione cronica di pazienti con frequenti episodi di scompenso acuto.

Parole chiave: Dialisi peritoneale incrementale, Scompenso cardiaco, Pazienti anziani.

Dichiarazione di conflitto di interessi: Gli Autori dichiarano di non avere conflitto di interessi.

Contributi economici degli autori: Gli Autori dichiarano di non aver ricevuto sponsorizzazioni economiche per la preparazione dell'articolo.

Indirizzo degli Autori:

Dr. Daniele Ciurlino

U.O. Nefrologia e Dialisi, Dipartimento Cardiovascolare IRCCS Multimedica

Via Milanese 300

20099 Sesto San Giovanni (MI)

daniele.ciurlino@multimedica.it

\section{Bibliografia}

1. De Vecchi AF, Scalamogna A, Finazzi S, Colucci P, Ponticelli C. Preliminary evaluation of incremental peritoneal dialysis in 25 patients. Perit Dial Int 2000; 20: 412-7.

2. KDOQI Clinical Practice Guidelines and Clinical Practice Recommendations for 2006 Updates: Hemodialysis adequacy, Pe- ritoneal Dialysis Adequacy and Vascular Access. Am J Kidney Dis 2006; 48 (Suppl. 1): S1-322.

3. Musetti C, Ciurlino D, Serra I, Bertoli SV. Low dose peritoneal dialysis as a first renal replacement therapy, Abstract presented at the World Congress of Nephrology 2009; Milan, Italy.

4. Bertoli SV, Musetti C, Ciurlino et al. Peritoneal ultrafiltration in refractory heart failure: a cohort study. Perit Dial Int. 2014 Jan;34: 64-70. 